\title{
Cloning, purification and characterization of the ribosomal protein L11 from E. coli
}

\author{
Roumiana Todorova
}

Institute of Biophysics and Biomedical Engineering, Bulgarian Academy of Sciences, Sofia, Bulgaria.

Email: todorova@,obzor.bio21.bas.bg

Received 15 March 2011; revised 20 March 2011; accepted 28 March 2011.

\begin{abstract}
A high-expression system of L11 was constructed and its interaction with other elements of the ribosome were investigated using physicochemical methods. The gene rplK, coding for the protein L11 from the $E$. coli $50 \mathrm{~S}$ ribosomal subunit was amplifyied, cloned and over-expressed. The protein L11 was purified under native and denaturing conditions, refolded and the structures of both proteins were compared. The protein L11 properly refolded from $6 \mathrm{M}$ urea after dialysis. Experiments on binding of proteins L11, RRF and EF-G from Escherichia coli were performed by analytical centrifugation and Biacore. Specific binding between protein L11 and RRF by analytical centrifugation was not detected probably due to structural reasons. These findings may be helpful in the design of new antibiotics that specifically disrupt the interactions in the "GTP-associated site" of the bacterial ribosome, as many of them are not effective anymore. A common intrinsically disordered region of protein L11 was found to be the amino acid sequence 86 - 97, while the residues 67 - 74, containing the linker region, are predicted to be disordered by DisEMBL.
\end{abstract}

Keywords: Cloning of L11; Rplk Gene; Purification under Denaturing Condition; Refolding; Interaction with Ribosome; Thiostrepton; Intrinsic Disorder

\section{INTRODUCTION}

The conserved ribosomal protein L11 of the large subunit of Escherechia coli plays a significant role during initiation, elongation and termination of protein biosynthesis. The protein L11 is important for the association of $30 \mathrm{~S}$ and $50 \mathrm{~S}$ ribosomal subunits and is involved in the factor-dependent hydrolysis of GTP in the peptydil-transferase center of the ribosome. The protein L11 binds to 23S RNA and is located on the surface of 50S subunit at the base of the stalk, composed from the com- plex (L7/L12) 4.L10. The N-terminal domain (NTD) of protein L11 is globular, situated at the surface of $50 \mathrm{~S}$ subunit, while the C-terminal domain (CTD) is involved in interaction with other ribosomal components [1]. The lysine methyltransferase PrmA post-translationally trimethylates the N-terminal alpha-amino and epsilonamino groups of Lys3 and Lys39, before the assembly of $50 \mathrm{~S}$ ribosomal subunit, thus stabilizing the protein L11 [2].

The ribosomal protein L11 is involved in the regulation of several ribosomal factor-dependent processes of the protein biosynthesis. The "GTP-associated site" that includes the L11-rRNA complex stimulates the GTPase activity of several ribosomal factors and is target for thiopeptide antibiotics thiostrepton and micrococcin. To clarify the regulation mechanism, a high-expression system of L11 was constructed and its interactions with other elements of the ribosome were investigated using physicochemical methods.

\section{MATERIALS AND METHODS}

\subsection{Cloning of L11}

The gene $r p l K$, coding for the ribosomal protein L11 from Esherichia coli was cloned from the chromosome of $E$. coli, strain DH5 $\alpha$. The designed primers, used for cloning of the gene rplK from the chromosome of E. coli strain DH5 $\alpha$, were: Primer1: 5' ATA TAG GAT CC T TA G TCC TC 3' and Primer2: 5' AGA TAG CAT ATG GCT AAG AA 3'.

One single colony from E.coli DH5 $\alpha$ was heated and mixed with 250 ng Primer1, 250 ng Primer2, 10x buffer PCR, dNTP mixure and 2U Taq DNA polymerase (Takara) for a 28 cycles of PCR. The purified PCR product and the vector $\mathrm{pET}-22 \mathrm{~b}+$ (Invitrogen) were digested with NdeI-BamHI and the fragments were loaded on $1.6 \%$ and $0.8 \%$ agarose gel respectively, cut from the gel, purified and ligated with Ligation mixure I (Takara).

The gene $r p l K$, coding for the $50 \mathrm{~S}$ ribosomal subunit protein L11 from E.coli, strain $\mathrm{DH} 5 \alpha\left(\mathrm{F}^{-} 80 \mathrm{~d} l a c Z\right.$ M15 
(lacZYA-argF) U169 recA1 endA1hsdR17 $\left(r_{k}^{-}, m_{k}^{+}\right)$ phoAsup 44 $^{-}$thi-1 gyr A96 relA1), was cloned in the poly-linker of the expression vector pET-22b+, between the sites of restriction BamHI and NdeI to obtain the recombinant plasmid pET-22b/L11. The obtained PCR product of $448 \mathrm{bp}$ was verified by DNA sequencing.

Minipreps of pET-22b/L11 were used for sequencing by PCR amplification on ABI Prism 310. The sequencing revealed the right sequence of the gene $r p l K$, coding for the ribosomal protein L11. The E. coli BL21 cells were transformed with $\mathrm{pET}-22 \mathrm{~b} / \mathrm{L} 11$ and induced with $1 \mathrm{mM}$ IPTG at $\mathrm{OD}_{550}=0.6$ for $3 \mathrm{~h}$ at $37^{\circ} \mathrm{C}$. The molecular weight of protein L11 was estimated with MW markers (Amersham Life Sciences).

\subsection{Purification of L11 under Native Conditions}

A single colony of $E$. coli BL21, transformed with pET-22b/L11, was grown in LB with Amp to $100 \mu \mathrm{g} / \mathrm{ml}$ at $37^{\circ} \mathrm{C}$ to $\mathrm{OD}_{550}=0.6$ and induced with IPTG to $1 \mathrm{mM}$ for $2.5 \mathrm{~h}$. The cells were harvested by centrifugation and washed with $20 \mathrm{mM}$ tris- $\mathrm{HCl}$, $\mathrm{pH} 7.8,20 \mathrm{mM} \mathrm{NH}_{4} \mathrm{Cl}$. The cells were resuspended in $20 \mathrm{mM}$ tris- $\mathrm{HCl}, \mathrm{pH} 7.6,50$ $\mathrm{mM} \mathrm{NH}_{4} \mathrm{Cl}, 10 \mathrm{mM} \mathrm{MgCl}, 0.8 \mathrm{mM} \mathrm{NaCl}, 7 \mathrm{mM} \beta$-ME, 1 mM PMSF. The cells were sonicated (with DNAse I), centrifuged at $16000 \mathrm{~g}$ for $10 \mathrm{~min}$, and again at $100000 \mathrm{~g}$ for $2 \mathrm{~h}$. The supernatant was loaded on a Superdex 75 column, equilibrated with $20 \mathrm{mM}$ tris- $\mathrm{HCl}, \mathrm{pH} 7.5,0.1 \mathrm{M}$ $\mathrm{NaCl}$ for gel-filtration. The fractions, containing L11 were loaded on DEAE-Sepharose or HiTrapQ column, equilibrated with $20 \mathrm{mM}$ tris- $\mathrm{HCl}, \mathrm{pH} 7.5,0.1 \mathrm{M} \mathrm{NaCl}$. The protein $\mathrm{L} 11$ was in the flow-through and after dialysis against $20 \mathrm{mM}$ tris- $\mathrm{HCl}, \mathrm{pH} 6.5,0.07 \mathrm{M} \mathrm{NaCl}$, was loaded on CM-Sepharose or HiTrap SP column in 0.07 $0.5 \mathrm{M} \mathrm{NaCl}$ gradient. The protein $\mathrm{L} 11$ was eluted at $0.1 \mathrm{M}$ $\mathrm{NaCl}$ in $20 \mathrm{mM}$ tris- $\mathrm{HCl}, \mathrm{pH} 6.5$, concentrated on Ultracel YM-3 and stored in $20 \%$ glycerol at $-80^{\circ} \mathrm{C}$. All purification procedures were accomplished at $4^{\circ} \mathrm{C}$.

\subsection{Purification of L11 under Denaturing Conditions}

The predominant part of the protein L11 was found insoluble in the cell debris. The protein L11 was solubilised in buffer $20 \mathrm{mM}$ tris-HCl, $\mathrm{pH}$ 6.5, $1 \mathrm{mM}$ DTT, 0.5 mM PMSF, 6M urea and after centrifugation at $16000 \mathrm{~g}$ was loaded on HiTrap SP for ion-exchange chromatography in gradient of $0-1 \mathrm{M} \mathrm{NaCl}$. The fractions with $\mathrm{L} 11$ were loaded on a column with Superdex 75, equilibrated with $20 \mathrm{mM}$ tris- $\mathrm{HCl}, \mathrm{pH}$ 7.5, $1 \mathrm{mM}$ DTT, 6M urea. An additional purification step was added on HiTrapQ in 20 $\mathrm{mM}$ tris- $\mathrm{HCl}, \mathrm{pH}$ 7.5, $1 \mathrm{mM}$ DTT, $6 \mathrm{M}$ urea and gradient of $0-1 \mathrm{M} \mathrm{NaCl}$. The purified protein $\mathrm{L} 11$ was renatured by dialysis against $30 \mathrm{mM}$ tris- $\mathrm{HCl}, \mathrm{pH} 7.9,20 \mathrm{mM}$ $\mathrm{MgCl}_{2}, 300 \mathrm{mM} \mathrm{KCl}$, concentrated on Centricon Ultracel
YM-3 and frozen in $20 \%$ glycerol at $-80^{\circ} \mathrm{C}$.

\subsection{Characterization of Protein L11}

The UV spectra of protein L11 were measured with a Jasco 530 spectrophotometer (JASCO). The CD spectra were measured with a Jasco J-720 CD spectrometer (JASCO) in the far UV region (184- $250 \mathrm{~nm}$ ) at $5 \mu \mathrm{M}$ $\mathrm{L} 11$ in $30 \mathrm{mM}$ tris- $\mathrm{HCl}, \mathrm{pH} 7.6,20 \mathrm{mM} \mathrm{MgCl} 2,300 \mathrm{mM}$ $\mathrm{KCl}$. Each spectrum recorded at $200-260 \mathrm{~nm}$ was repeated twice.

\section{RESULTS AND DISCUSSIONS}

\subsection{Cloning and Purification of Protein L11 from $E$. coli}

The obtained PCR product was the gene $r p l K$, coding for the protein L11 from the 50S ribosomal subunit of $E$. coli strain DH5 $\alpha$. The sequencing revealed the rplK gene analogous to that reported in [GenBank: BAE77337.1, NCBI Database] and [NP_290614, NCBI Database]. The translated amino acid sequence of the protein L11 from the gene sequencing, used also in the predictions, was:

50S Ribosomal Protein L11 from E. coli

MAKKVQAYVK LQVAAGMANP SPPVGPALGQ QGVNIMEFCK AFNAKTDSIE KGLPIPVVIT VYADRSFTFV TKTPPAAVLL KKAAGIKSGS GKPNKDKVGK ISRAQLQEIA QTKAADMTGA DIEAMTRSIE GTARSMGLVV ED

The expression of protein L11 in Escherichia coli was induced by IPTG (Figure 1). The molecular weight of protein L11 was estimated with MW markers on SDS-PAGE and later confirmed by gel-filtration. The cloned protein L11 was composed from 142 AA with calculated MW of $14875 \mathrm{Da}$. The soluble part of protein L11 in the cell extract was purified in native conditions (Figure 2). The predominant part of protein L11, found insoluble in the cell debris, was used for purification in denaturing conditions (6M urea). The yield of the puri

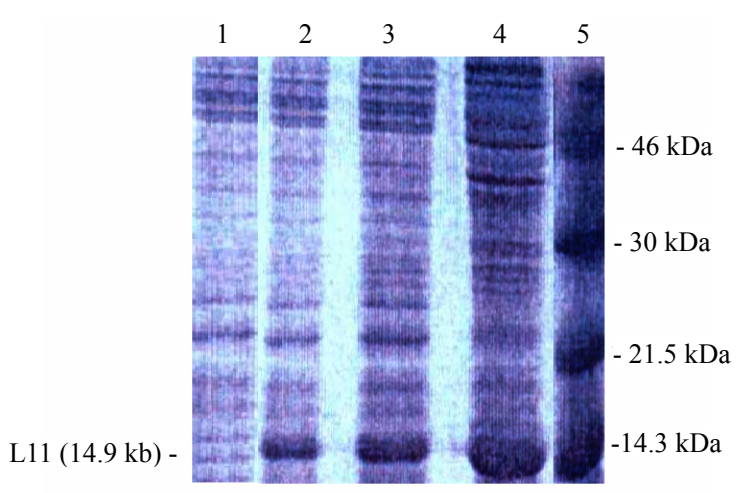

Figure 1. Expression of the protein L11 in E. coli, induced with IPTG. $1.1 \mu \mathrm{l}$ of cells $E$. coli B121 w.t.; $2.1 \mu \mathrm{l}$ of BL21 cells expressing L11.; $3.2 \mu$ of cells expressing L11.; 4 . $3 \mu$ of cells expressing L11.; 5. MW markers. 
L11 purification protocol

Cells (E. coli + pET22b/L11 + IPTG)

$\downarrow$

Sonication

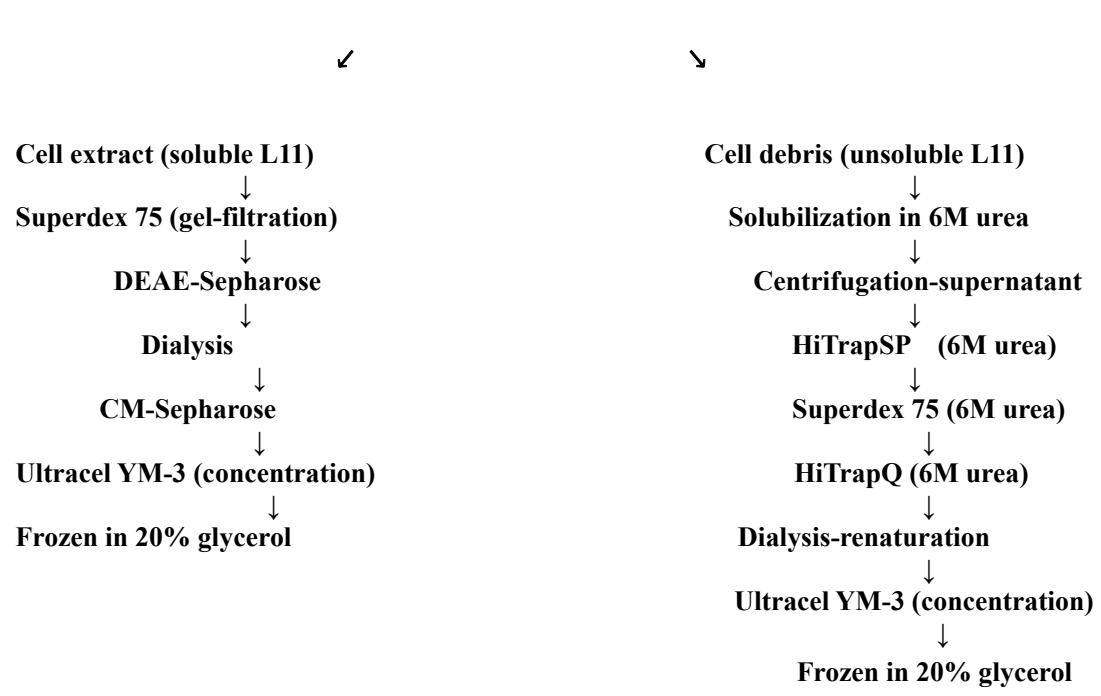

Figure 2. Purification protocol for the ribosomal protein L11 from E. coli in native and denaturing conditions.

fied protein L11 in denaturing conditions was about twice comparing to the yield, obtained in native conditions, due to the high level of unsolubility and hydrophobicity (Figures 3(a), (b)). The methylation (at positions 1, 3 and 39) does not affect the mobility of the protein L11 on the SDS-PAGE, because all the protein is moving in the same band (during the purification), but slowly than expected, estimated by the MW marker. The protein L11 is stable at $\mathrm{pH}$ close to 9.0 and does not aggregate at relatively low concentrations (pI of 10.8).

During purification procedure the protein L11 was eluted as one single peak from non-denaturing conditions and from urea-containing buffers. The method of renaturation (dilution, dialysis or ultrafiltration) does not affect the stability of protein L11, but the lower concentration was important for properly refolding, allowing form intramolecular interactions, and decreasing the intermolecular interactions. During the L11 purifying procedure in native conditions the elution peak, monitored at $254 \mathrm{~nm}$ was much more intensive than that at $280 \mathrm{~nm}$ that can be a sign of nucleic acid binding (an RNA binding protein). In denaturing conditions (6 M urea) the purification profile of L11 was as usually for protein purification (at 280, 264 and $215 \mathrm{~nm}$ ).

The far-UV spectra show that the recombinant form often does not have the same secondary structure as the natural protein. The UV and CD spectra of protein L11, isolated in native and in denaturing conditions was of monomeric molecules and did not differ (protein is properly refolded). Thus the folding of the protein (Figure 4) was not affected from the denaturation-renaturation procedure under the conditions used: low concentration of protein L11 (about or under $1 \mathrm{mg} / \mathrm{ml}$ ), temperature of $4^{\circ} \mathrm{C}$ during all procedures and relatively high salt concentration (above $0.3 \mathrm{M}$ ), resulting in the complete renaturation of protein L11 after dialysis. The obtained CD spectrum of L11 in the range $200-260 \mathrm{~nm}$ (far UV region) has one minimum at $208 \mathrm{~nm}$ and a shoulder at $220 \mathrm{~nm}$. The presence of a single minimum in the range $210-220 \mathrm{~nm}$ is corresponding to some content of $\beta$-strand in the secondary structure and limited content of helix, similar to the predicted secondary structure on Figure 5. The crystal structure of protein L11 was determined in complex with stabilizing fragments of rRNA or in the whole ribosome, where the protein is participating in multiple interactions with other elements of the ribosome and is functionally active in vivo and in vitro. Even on the crystal structure of the $E$. coli ribosome were found large unstructured regions in L11, very flexible and situated at the most flexible part of the ribosome. This is related with the function of L11 on the bacterial ribosome.

The results from the sedimentation of the native protein L11 show a higher molecular weight than the calculated, due probably to the higher dimensions and the asymmetric shape of the molecule in solution, reflecting the incompact structure and high unstructured regions. At low concentration the protein L11 does not selfassociate and aggregate, and is stable in solution. The $\alpha$-helix content of native protein L11, isolated from $50 \mathrm{~S}$ sub-particle of $E$. coli ribosomes was estimated to be 


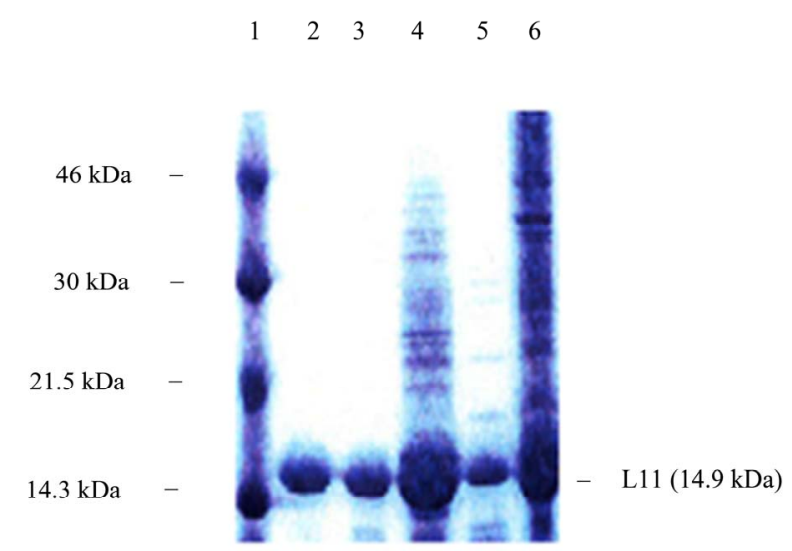

(a)

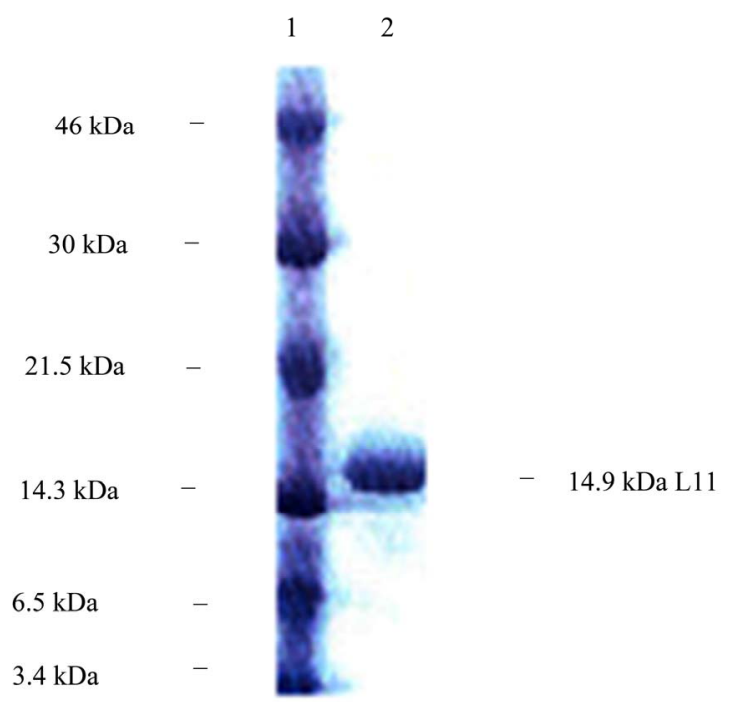

(b)

Figure 3. Purification of protein L11. (a) Purification of protein L11 under denaturing conditions: 1. MW markers; 2. Purified protein L11 after renaturation by dyalisis; 3 . Purified protein L11 from the anion-exchange column HiTrapQ; 4. Flow-through (fractions) from the cation-exchange column HiTrap SP; 5. Fractions, containing protein L11 from the gel-filtration column Superdex 75; 6. Cell-debris, containing insoluble protein L11; (b) Purified protein L11 under native conditions: 1. MW markers $(46.0,30.0,21.5,14.3,6.5,3.4$, $2.35 \mathrm{kDa}) ; 2$. Purified native protein L11.

about 37\% [3]. The results revealed that the protein L11 was properly refolded from urea and has a tertiary structure. The increasing of the ionic strength of the buffer is increasing the solubility of the protein and its stability in solution. The results on equilibrium sedimentation of ribosomal protein L11, together with EF-G and RRF shown that the proteins, two by two and all together does not form complexes in free form spontaneously or they are considered to be unspecific. From the sedimentation the MW of the particles (estimated to be $34.6 \mathrm{kDa}$ ) corresponded to the sum of the MW of L11 (about 14.9

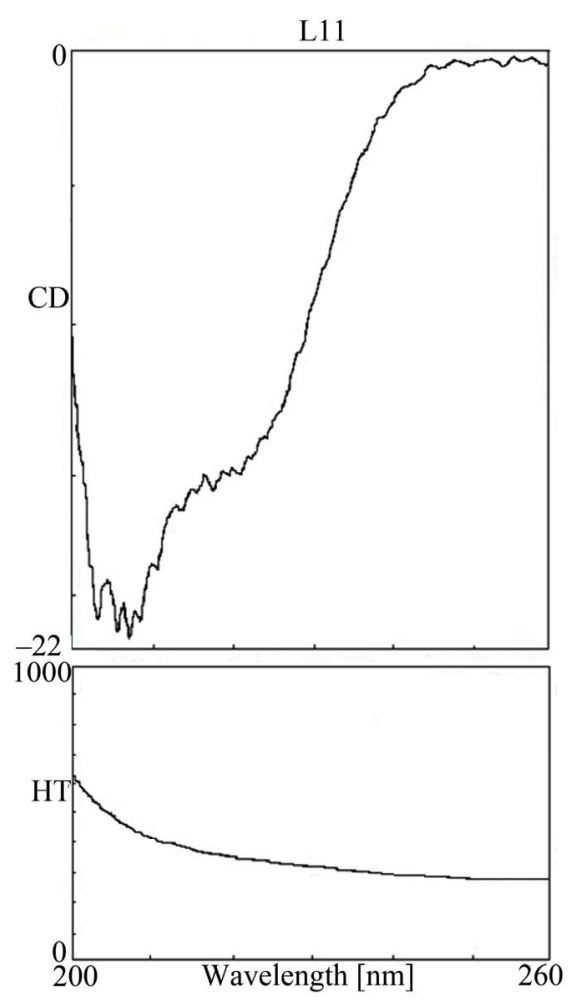

Figure 4. The CD spectrum of the refolded protein L11 was measured with a Jasco J-720 CD spectrometer (JASCO) in 0.1 $\mathrm{mm}$ cell in the far UV region $(200-260 \mathrm{~nm})$ at final concentration into the cell of $5 \mu \mathrm{M}$ L11 in buffer $30 \mathrm{mM}$ tris- $\mathrm{HCl}, \mathrm{pH}$ 7.6, $20 \mathrm{mM} \mathrm{MgCl}_{2}, 300 \mathrm{mM} \mathrm{KCl}$ and $25^{\circ} \mathrm{C}$.

$\mathrm{kDa}$ ) and RRF (about $20 \mathrm{kDa}$ ) (Figure 7). The protein L11 has a big affinity to the support when it was subject to immobilization on BIACORE, considered to be unspecific. The direct assays on Biacore between RRF and L11 (when RRF was immobilized) showed high binding of L11 that was considered to be non-specific.

Some physicochemical characteristics of ribosomal protein L11 were determined on the protein, isolated from native $E$. coli ribosomes $[1,4,5]$. The obtained structural data in this study, were the ribosomal protein L11 was over-expressed in E. coli and purified in non-denaturing and denaturing conditions, are similar to that reported before for L11, directly purified from $E$. coli ribosomes. The over-expressed protein L11 is folding properly and the presence of N-terminal Met in the cloned protein (removed in the endogenous protein L11 from the native ribosomes) and the big concentration difference between the cytoplasm (where L11 is in soluble form), the including bodies (where L11 is in insoluble form) and the ribosomes (where L11 is in bound form; in E. coli it was about $10 \mu \mathrm{g} / \mathrm{ml}$ [5]) does not affect the structure of protein. At temperatures higher than $37^{\circ} \mathrm{C}$ the protein L11 is aggregating because of denaturation, increasing with the concentration of protein and 
decreasing with the salt concentration up to $1 \mathrm{M}$. The reported thermal denaturation of protein L11 showed low thermostability with a transition of about $50^{\circ} \mathrm{C}$. The aggregation appeared after the middle of the transition, but the existence of a peak is evidence of a cooperative structure [6]. The process was irreversible and an enthalpy of denaturation could not be calculated. The high level of secondary structure and the shape of the thermal denaturation curve are evidence for the existence of regions of tertiary structure in the protein L11 [4]. The structure of protein L11 is stabilized in the whole ribosome or in the complex with other ribosomal proteins and $23 \mathrm{~S}$ rRNA.

\subsection{Prediction of Secondary Structure of Protein L11 in Solution}

The structure of proteins L11 in solution was predicted using the protein structure prediction programs PSIPRED [http://bioinf3.cs.ucl.ac.uk/cgi-bin/psipred] [7], SOSUI [http://bp.nuap.nagoya-u.ac.jp/sosui], TMPRED [http://www.ch.embnet.org], PSOFT [http://psort.ims.u- tokyo.ac.jp], PredictProtein [http://www.predict-protein. org].

The average hydrophobicity, estimated for protein L11 by SOSUI was $(-0.061268)$, corresponding to a soluble cytoplasmic protein, with no N-terminal signal peptide. The PSIPRED prediction results for protein L11 in solution [http://bioinf3.cs.ucl.ac.uk/cgi-bin/ psipred] are shown in Figure 5. The prediction parameters for break point for protein L11 in solution shown that some of them have functional implications such as Pro74 (taking part in the flexible unstructured region) and Pro22 (situated in the proline-rich helix of L11).

In result the predicted secondary structure of protein L11 in solution is close to the structure, suggested on Figure 4 and in the complexes with 23S rRNA.

The SDS-PAGE revealed the expression of the ribosomal protein L11 with size of $14.9 \mathrm{kDa}$, estimated with MW markers. The hydrodynamic parameters, obtained from gel-filtration and sedimentation also help determine the degree of the polypeptide chain compaction. Two

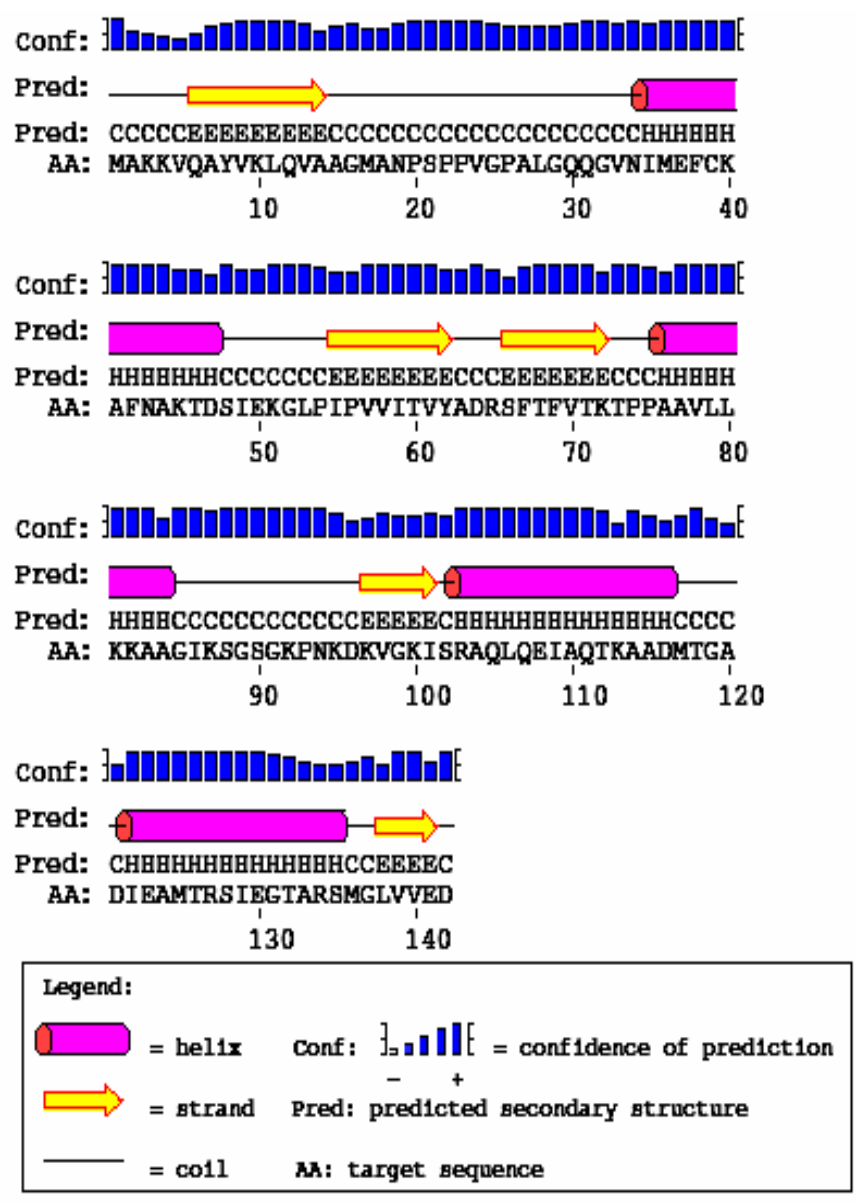

Figure 5. PSIPRED prediction results for the protein L11 from E. coli in solution [http://bioinf3.cs.ucl.ac.uk/cgi-bin/psipred]. 


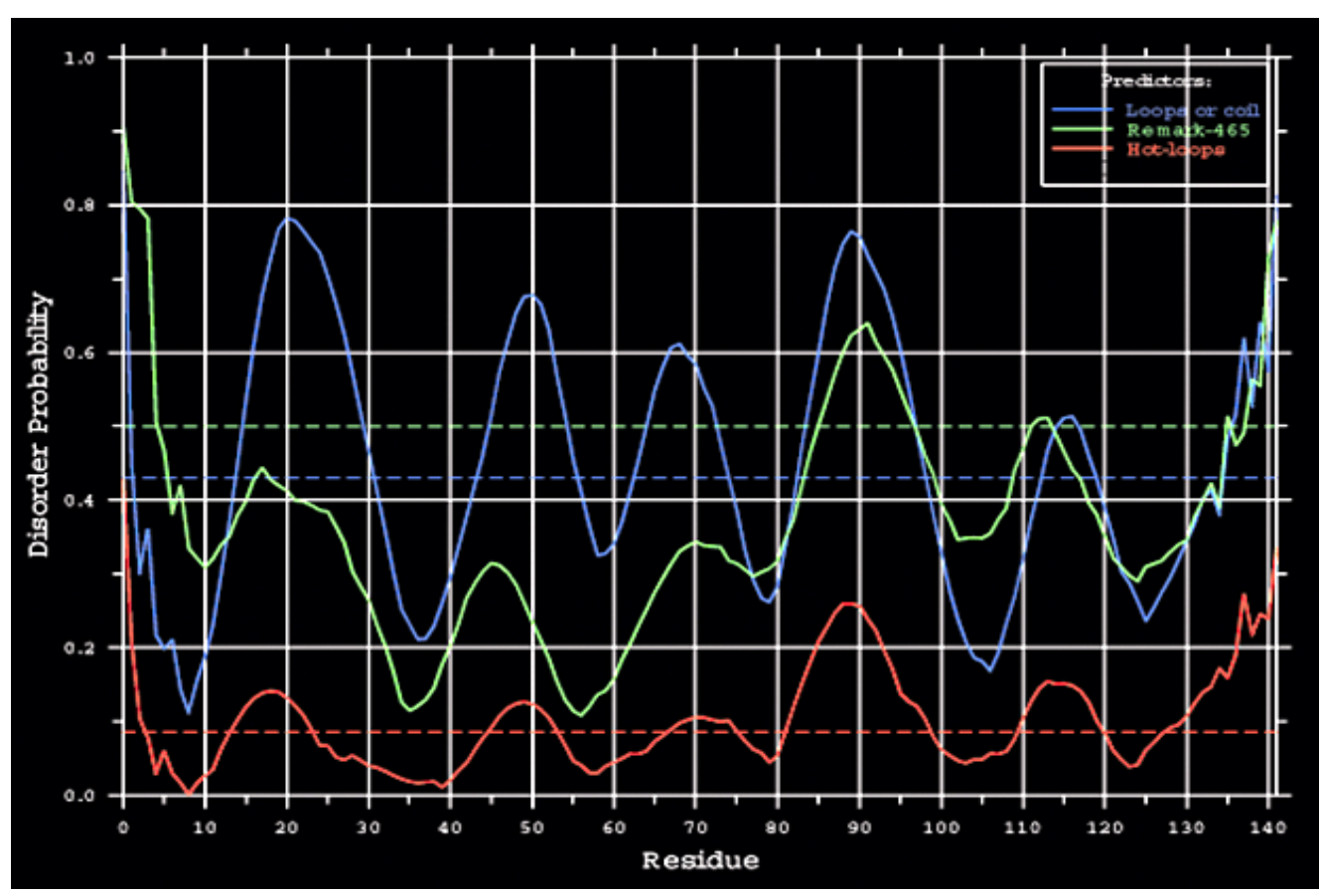

(a)

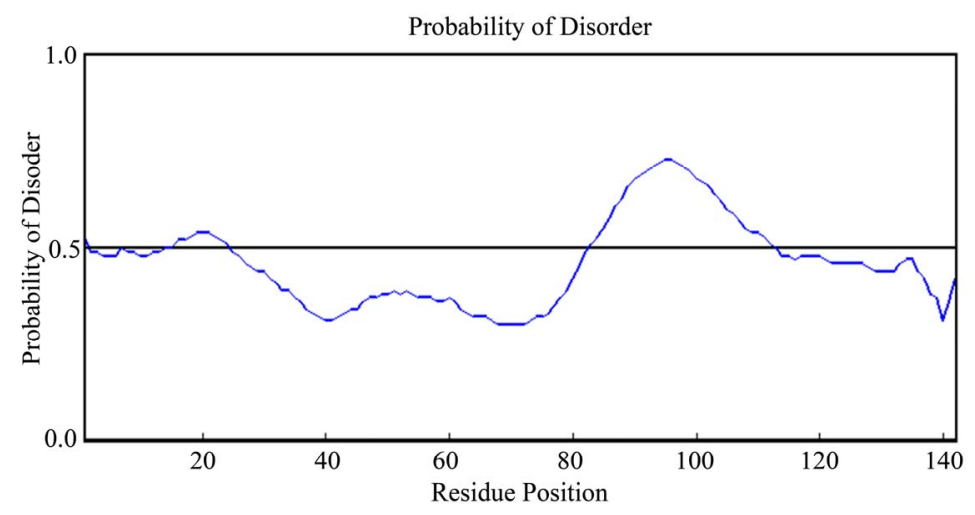

(b)

Figure 6. Protein disorder prediction for the ribosomal protein L11: (a) DisEMBL Intrinsic Protein Disorder Prediction of protein L11 from E. coli [http://dis.embl.de/cgiDict.py] [31]; (b) Disordered amino-acid regions of L11 by RONN [http://www.strubi.ox.ac.uk/RONN] [34].

such regions with essential disorder are detected in the isolated ribosomal protein L11 from the large subunit of the ribosome: the amino acid residues $1-10$ and $18-34$ have no fixed structure by the NMR data of L11-C76 of B. stearothermophilus ribosomal protein L11 $[8,9]$. The ribosomal proteins in general lack a well defined structure when separated from rRNA $[10,11]$. Some proteins must be unfolded or disordered in order to perform their functions, and others fold only in complex with target structures. The intrinsically disordered proteins play roles in processes such as protein-RNA recognition, in the example of the ribosomal proteins L11-C76 from $B$. stearothermophilus L11 [12].

\subsection{Prediction Results for the Protein L11 Intrinsic Disorder}

The disordered segments of L11-C76 correspond to the amino acid sequences in the whole B. stearothermophilus protein L11 (AA residues 71 - 74 and 85 - 100), and to the L11 from E. coli (AA residues 72 - 75), containing the absolutely conserved region KTPP in all bacteria, and (AA residues 85 - 98), conserved in all bacteria, especially the $\mathrm{P}, \mathrm{AG}, \mathrm{GS}, \mathrm{KV}$. The disordered segments of L11 were confirmed by the prediction programs DisEMBL, IUPRED (partially), FoldIndex, RONN and PrDOS (partially) for the amino acid region (AA resi- 


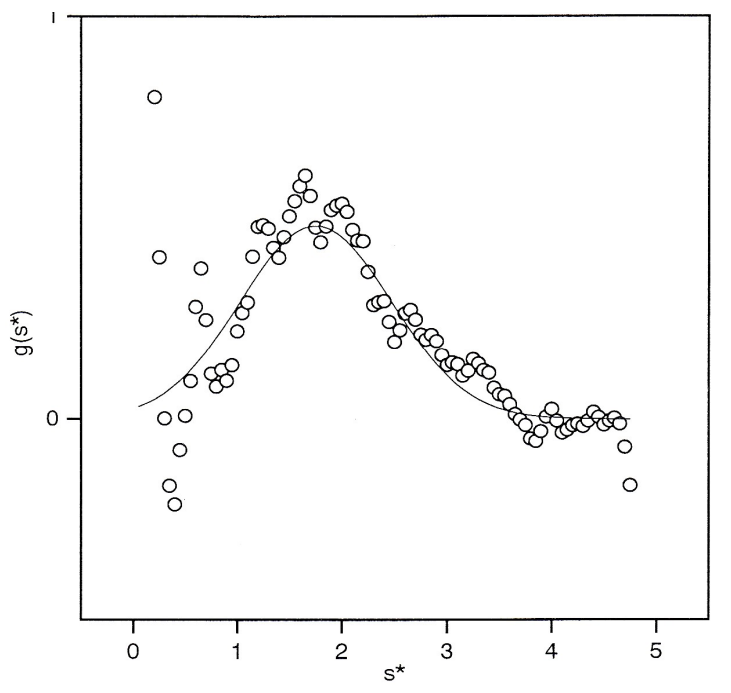

Figure 7. Sedimentation (time-derivative analysis). Plot of $\mathrm{g}\left(\mathrm{s}^{*}\right)$ versus $\mathrm{s}^{*}$ for the mixture of L11 and RRF. Speed 50,000, S 1.75 and estimated MW 34591.5.

dues 85 - 98). The L11 protein disorder was predicted by the Predictors PONDR VL3, IUPRED, RONN, FOLD INDEX [http://www.disprot.org/predictors.php] and the different methods given partially different results. The obtained disordered AA regions were:

I. DisEMBL Intrinsic Protein Disorder Prediction 1.5. [http://dis.embl.de/cgiDict.py] [13]:

Disordered AA residues by Loops/coils definition: none LOPS 15 - 31, 45 - 56, 64 - 74, 84 - 99. Disordered AA residues by Hot-loops definition: none HOTLOOPS 15 - 24, 46 - 54, 82 - 99, 111 - 120, 129 - 142. Disordered AA residues by Remark-465 definition: none REM465 86-97. (Figure 6(a)).

II. IUPRED Prediction of Intrinsically Unstructured Proteins [http://iupred.enzim.hu/index.Html][14]:

Position Residue Disorder - Long disorder - Disorder tendency (score above 0.5) at AA 20 - 26, 91 - 103, 124 $-126$. [15].

III.FoldIndex. [http://bip.weizmann.ac.il/fldbin/findex]

Unfoldability 0.173 (Charge: 0.049, Phobic: 0.493).

Predicted disorder AA segment: [85]-[90] length: 6 score: $-0.04 \pm 0.03$.

Predicted disorder AA segment: [92]-[96] length: 5 score: $-0.02 \pm 0.01$.

Predicted disorder AA segment: [102]-[106] length: 5 score: $-0.02 \pm 0.02$.

IV. RONN (Regional Order Neural Network). [http://www.strubi.ox.ac.uk/RONN] [16]:

Disordered AA regions of L11: AA 1 - 1, 16 - 24, 83 112 (Figure 6(b)).

A common disordered region was the amino acid sequence (AA residues 86 - 97) (Figures 6(a), (b)). The
AA fragment (67 - 74), containing the linker region, was predicted to be disordered by DisEMBL.

\subsection{Structure and Function of the Complex L11-23S RNA}

The comparative study of protein L11, obtained under denaturing and native conditions did not show differences in the CD and UV spectra. The partial unsolubility of the protein and its hydrophobicity can be explained with the biological role of L11 to participate in complex formation with $23 \mathrm{~S}$ rRNA, L10 and the L7/L12 tetramer at the stalk of the 50S ribosome, resulting in the functional complex L11.L10.(L7/L12) 4 . Thus L11 is stabilized by the complex formation, changing the conformation upon participating in the movement and binding to the ribosomal factors. The GTPase domain of 50S ribosomal subunit of Escherichia coli is composed from the ribonucleoprotein complex L11-23S rRNA and the adjacent pentameric complex L10. (L7/L12) 4 .

The suggested role of L11 in recycling was the trigger for in vitro experiments on binding of L11 to EF-G and RRF by sedimentation and BIACORE. The experiments on binding of proteins L11, RRF and EF-G from Escherichia coli were performed by analytical centrifugation. Specific binding between these proteins was not detected that can be explained with structural reasons. Most probably the whole ribosome is required for specific interactions between these proteins. The direct assays on Biacore with immobilised RRF and purified L11 from $E$. coli in the flow trough have shown a high binding of L11 that was considered to be non-specific. These findings may be due to the presence of unfolded regions in the L11 molecule in solution and its high hydrophobicity. The crystal structure of L11 was solved in complex with fragments of $23 \mathrm{~S}$ rRNA that bind to and stabilizes the protein [17]. The protein L11 has a high intrinsic propensity to aggregate and extensively uses disordered regions to mediate and modulate interactions with other proteins. This is related to the function of L11 in the ribosome and the binding of multiple sites by multiple interactions with RNA and other ribosomal proteins in the formation of RNA-protein complexes.

\subsection{Unfolding-Structure and Translational Regulation via L11}

Protein-protein and protein-nucleic acid interactions are central to the ribosome and involve coupled folding and binding of the partners. The high specificity and low affinity of intrinsic disorder interactions with functional partners is very important for transient protein-protein and protein-nucleic acid interactions [18].

Ribosomal protein L11 consists of two globular domains connected by a linker region. There is some flexi- 
bility between the two domains, but the linker is short and contains two conserved Pro (73 and 74) that provide rigidity and conserved hydrophobic residues Met-52, Ile-53, Pro-55, Pro-73, and Phe-77. The structure of the L11 NTD consists of two helices packed against an antiparallel $\beta$-sheet, with an overall $\beta 1-\alpha 1-\alpha 2-\beta 2-\beta 3$ topology. The $\mathrm{N}$-terminal seven residues are disordered and the Pro-rich helix 1 appears to have a crucial functional role. The CTD domain consists of an overall $\alpha 3-\beta 4-\alpha 4-\alpha 5-\beta 5$ topology and contributes to a conserved hydrophobic core. The domain is characterized by two extended loops that are disordered in the absence of the RNA but have defined structures in the complex [17]. The CTD binds RNA tightly while the NTD makes only limited contacts with rRNA functioning as a switch [17]. The CTD of L11 uses the homeodomain helix to bind RNA and the NTD $\alpha$ PLUSPUSSIGN $\beta$ fold is similar to other RNA-binding proteins. It also reveals novel principles of RNA folding, RNA-protein recognition, and indirect RNA tertiary structure stabilization. The NTD functions as a molecular switch, either by facilitating changes in the GAR RNA or by controlling access to the RNA. Thiostrepton and micrococcin bind to the NTDRNA interface, thus locking the switch and disrupting factor function [17]. They target both L11 as well as $23 \mathrm{~S}$ rRNA. Thiostrepton cannot bind free L11 and binds naked 23S rRNA with affinity lower (about $10^{3}$ ) than the intact ribosome. Resistance to thiostrepton and micrococcin can be obtained by deletion or mutations in the rplK gene encoding L11. Micrococcin is a stimulator of the GTPase activities of EF-G while thiostrepton is a translocation inhibitor preventing the EF-G-catalyzed movement of tRNAs on the ribosome. Both thiostrepton and nosiheptide stabilize the structure of L11-NTD, inducing a conformational change that perturbs the L11-L7 interaction. Micrococcin may function by stabilizing the L11-L7 interaction and thus pre-positioning L7 on L11 so that it can interact with EF-G. The double-switching mechanism identified in L11 acts as a molecular sensor to monitor and control the translational state of the ribosome and may be universal [19]. Thus the protein L11 acts as a molecular switch to control L7 binding and regulate EF-G turnover during protein synthesis [19]. Binding of thiostrepton results in more rigid conformations near the linker (Thr71) and near its putative binding site (Leu12), involving residues in the 3(10)-helix in L11 [20].

The protein L11 is composed from a dynamic NTD (AA residues 1 - 72) connected to the RNA-binding CTD (AA residues 76 - 142) by a flexible linker (AA residues 73 - 75). In vivo the mutation of $\mathrm{P} 22$ in the NTD of L11 is important for initiation of the stringent response, while the removal of L11, the linker and the proline-rich helix resulted in deactivation [21]. The L11 enhances mRNA and rRNA binding to L10. (L12) 4 about 100-fold [22]. The CTD of L11-C76 recognizes and binds to a conserved 58 nd of $23 \mathrm{~S}$ rRNA. The Loop 1 was flexible and disordered for L11-C76 free in solution, while in the RNA-bound form it is rigid and adopts a specific conformation [8]. The NTD residues $11,12,24$, $28,29,33,36$ and 56 were active restraints to either thiostrepton or the RNA (1059 - 1064, $1068-1070$ and 1095 - 1098). Residues 9 - 14, 21 - 37, 39, 40, 53, 55 57,74 of L11 were passive restraints for the interaction with thiostrepton. The interaction with rRNA influences the dynamical properties of both L11 domains [23]. Binding of RNA to L11 induces rigidification of a flexible loop in the CTD and reorientation of the NTD. The domain orientation in free L11 shows limited similarity to that of ribosome-bound L11 in complex with EF-G [24]. The NTD of L11, the CTD of L7/L12, and the G' domain of EF-G participate in formation of an arc-like connection [25]. The rRNA stabilized by the CTD of L11 stabilizes EF-G, binding in the post-translocation state [26]. The initial factor binding to the ribosome results in a movement of L11 NTD toward 50S subunit. After GTP hydrolysis this domain moves away from the 50S subunit to make complex with EF-G and GDP, and finally the NTD of L11 returns to its resting position. The L11 NTD may occupy a wide range of positions, relative to the 50S subunit [27]. RRF may bind close to the GTPase domain [28,29], the complex L11-rRNA and EF-G in the recycling. In an RRF-70S complex was observed a site-specific cross-linking from Cys39 of E. coli L11 to Tyr45 within domain II of E. coli RRF [29].

The experiments on binding of proteins L11, RRF and EF-G from E. coli, performed by analytical centrifugation and Biacore did not reveal specific binding between these proteins. Because of the high aggregation tendency of L11 in solution, non-stabilized by complex formation as in vivo, specific interactions could not be detected. Other experiments can be designed and completed, concerning complexed form of L11, especially with fragments of 23S RNA, and the adjacent pentameric complex L10.(L12) $)_{4}$ from the stalk of the ribosome.

\section{CONCLUSIONS}

The folding of protein L11, purified in native and non-denaturing conditions is essentially identical. Specific binding between protein L11, EF-G and RRF by analytical centrifugation was not detected because of structural reasons. The direct assays on Biacore between RRF and L11 show a high binding of L11 that was considered to be non-specific because of high hydrophobicity of L11 and the intrinsic disorder of the molecule. 
These findings can be taken in consideration designing new antibiotics that specifically disrupt the interactions in the "GTP-associated site" of the bacterial ribosome, as many of them are not effective anymore because of bacterial resistance against them. A common intrinsically disordered region of protein L11 was found to be the amino acid sequence 86 - 97.

\section{ACKNOWLEDGEMENTS}

The author would like to thank H. Shimahara and H. Uchiyama from the Graduate School of Pharmaceutical sciences, Osaka University for the help and the discussions.

\section{REFERENCES}

[1] Choli, T. (1989) Structural properties of ribosomal protein L11 from Escherichia coli. Biochemistry International, 19, 1323-1338.

[2] Demirci, H., Gregory, S.T., Dahlberg, A.E. and Jogl, G. (2007) Recognition of ribosomal protein L11 by the protein trimethyltransferase PrmA. The EMBO Journal, 26, 567-77. doi: $10.1038 /$ sj.emboj. 7601508

[3] Gudkov, A.T. and Tumanova, L.G. (1979) A physicchemical characteristic of ribosomal proteins L10 and L11. Molecular Biology, 14, 798-802.

[4] Tumanova, L.G., Gudkov, A.T., Bushuev, V.N. and Okon, M.S. (1981) Study of the tertiary structure of protein L11 from Escherichia coli ribosomes in solution by proton magnetic resonance. FEBS Letters, 127, 241-244. doi:10.1016/0014-5793(81)80215-3

[5] Kime, M.J., Tatcliffe, R.G., Moore, P.B. and Williams, R.J.P. (1980) On the renaturation of ribosomal protein L11. European Journal of Biochemistry, 110, 493-498. doi:10.1111/j.1432-1033.1980.tb04891.x

[6] Khechinashvili, N.N., Koteliansky, V.E., Gogia, Z.V., Littlechild, J. and Dijk, J. (1978) A heat denaturation study of several ribosomal proteins from Escherichia coli by scanning microcalorimetry. FEBS Letters, 95, 270-272. doi:10.1016/0014-5793(78)81008-4

[7] McGuffin, L.J., Bryson, K. and Jones, D.T. (2000) The PSIPRED protein structure prediction server. Bioinformatics, 16, 404-405.

doi:10.1093/bioinformatics/16.4.404

[8] Hinck, A.P., Markus, M.A., Huang, S., Grzesiek, S., Kustonovich, I., Draper, D.E. and Torchia, D.A. (1997) The RNA binding domain of ribosomal protein L11: Three-dimensional structure of the RNA-bound form of the protein and its interaction with 23S rRNA. Journal of Molecular Biology, 274, 101-113. doi:10.1006/jmbi.1997.1379

[9] Serdyuk, I.N. (2007) Structured proteins and proteins with intrinsic disorder. Molecular Biology, 41, 262-277.

[10] Dunker, A.K., Brown, C.J., Lawson, J.D., Iakoucheva, L.M. and Obradovic, Z. (2002) Intrinsic disorder and protein function. Biochemistry, 41, 6573-6582. doi:10.1021/bi012159+

[11] Uversky, V.N., Gillespie, J.R. and Fink, A.L. (2000) Why are "natively unfolded" proteins unstructured under physiologic conditions? Proteins, 41, 415-427.
doi:10.1002/1097-0134(20001115)41:3<415::AID-PROT 130>3.0.CO;2-7

[12] Markus, M.A., Hinck, A.P., Huang, S., Draper, D.E. and Torchia, D.A. (1997) High resolution solution structure of ribosomal protein L11-C76, a helical protein with a flexible loop that becomes structured upon binding to RNA. Nature Structural Biology, 4, 70-77. doi:10.1038/nsb0197-70

[13] Linding, R., Jensen, L.J., Diella, F., Bork, P., Gibson, T.J. and Russell, R.B. (2003) Protein disorder prediction: Implications for structural proteomics. Structure, 11, 1316-1317.

[14] Dosztanyi, Z., Csizmok, V., Tompa, P. and Simon, I. (2005) IUPred: Web server for the prediction of intrinsically unstructured regions of proteins based on estimated energy content. Bioinformatics, 21, 3433-3434. doi:10.1093/bioinformatics/bti541

[15] Prilusky, J., Felder, C.E., Zeev-Ben-Mordehai, T., Rydberg, E.H., Man, O., Beckmann, J.S., Silman, I. and Sussman, J.L. (2005) FoldIndex: A simple tool to predict whether a given protein sequence is intrinsically unfolded. Bioinformatics, 21, 3435-3438. doi:10.1093/bioinformatics/bti537

[16] Yang, Z.E., Thomson, R., McNeil, P. and Esnouf, R.M. (2005) RONN: The bio-basis function neural network technique applied to the detection of natively disordered regions in proteins. Bioinformatics, 21, 3369-3376. doi:10.1093/bioinformatics/bti534

[17] Wimberly, B.T., Guymon, R., McCutcheon, J.P., White, S.W. and Ramacrishnan, V. (1999) A detailed view of a ribosomal active site: The structure of the L11-RNA complex. Cell, 97, 491-502. doi:10.1016/S0092-8674(00)80759-X

[18] Jiangang, L., Narayanan, B.P., Christopher, J.O., Eric, W.S., Uversky, V.N. and Dunker, A.K. (2006) Intrinsic disorder in transcription factors. Biochemistry, 45, 6873-6888. doi:10.1021/bi0602718

[19] Harms, M., Wilson, D.N., Schluenzen, F., Connell, S.R., Stachelhaus, T., Zaborowska, Z., Spahn, C.M. and Fucini, P. (2008) Translational regulation via L11: Molecular switches on the ribosome turned on and off by thiostrepton and micrococcin. Molecular Cell, 30, 26-38. doi:10.1016/j.molcel.2008.01.009

[20] Lee, D., Walsh, J.D., Yu, P., Markus, M.A., Choli-Papadopoulou, T., Schwieters, C.D., Krueger, S., Draper, D.E. and Wang, Y.X. (2007) The structure of free L11 and functional dynamics of L11 in free, L11 rRNA(58 nt)binary and L11-rRNA(58 nt)-thiostrepton ternary complexes. Journal of Molecular Biology, 367, 1007-1022. doi:10.1016/i.jmb.2007.01.013

[21] Jenvert, R.M. and Schiavone, L.H. (2007) The flexible $\mathrm{N}$-terminal domain of ribosomal protein L11 from Escherichia coli is necessary for the activation of stringent factor. Journal of Molecular Biology, 365, 764-772. doi:10.1016/j.jmb.2006.10.065

[22] Iben, J.R. and Draper, D.E. (2008) Specific interactions of the L10.(L12)4 ribosomal protein complex with mRNA, rRNA, and L11. Biochemistry, 47, 2721-2731. doi:10.1021/bi701838y

[23] Jonker, H.R., Ilin, S., Grimm, S.K., Wöhnert, J. and Schwalbe, H. (2007) L11 domain rearrangement upon binding to RNA and thiostrepton studied by NMR spec- 
troscopy. Nucleic Acids Research, 35, 441-454. doi:10.1093/nar/gk11066

[24] Ilin, S., Hoskins, A., Ohlenschläger, O., Jonker, H.R., Schwalbe, H. and Wöhnert, J. (2005) Domain reorientation and induced fit upon RNA binding: Solution structure and dynamics of ribosomal protein L11 from Thermotoga maritime. A European Journal of Chemical Biology, 6, 1611-1618. doi:10.1002/cbic.200500091

[25] Datta, P.P., Sharma, M.R., Qi, L., Frank, J. and Agrawal, R.K. (2005) Interaction of the G' domain of elongation factor $\mathrm{G}$ and the $\mathrm{C}$-terminal domain of ribosomal protein L7/L12 during translocation as revealed by cryo-EM. Molecular Cell, 20, 723-731. doi:10.1016/j.molcel.2005.10.028

[26] Bowen, W.S., Van Dyke, N., Murgola, E.J., Lodmell, J.S. and Hill, W.E. (2005) Interaction of thiostrepton and elongation factor-G with the ribosomal protein L11-binding domain. Journal of Biological Chemistry, 280, 2934-2943. doi:10.1074/jbc.M407008200

[27] Kavran, J.M. and Steitz, T.A. (2007) Structure of the base of the L7/L12 stalk of the Haloarcula marismortui large ribosomal subunit: Analysis of L11 movements. Journal of Biological Chemistry, 371, 1047-1059. doi:10.1016/j.jmb.2007.05.091

[28] Todorova, R.T. and Saihara, Y. (2003) Specific binding of ribosome recycling factor (RRF) with the Escherichia coli ribosomes by BIACORE. Molecular Biology Reports, 30, 113-119. doi:10.1023/A:1023991026045

[29] Wilson, D.N., Schluenzen, F., Harms, J.M., Yoshida, T., Ohkubo, T., Albrecht, R., Buerger, J., Kobayashi, Y. and Fucini, P. (2005) X-ray crystallography study on ribosome recycling: The mechanism of binding and action of RRF on the 50S ribosomal subunit. The EMBO Journal, 24, 251-260. doi:10.1038/sj.emboj.7600525 